\title{
Cytokine Combination Therapy with Erythropoietin and Granulocyte Colony Stimulating Factor in a Porcine Model of Acute Myocardial Infarction
}

\author{
Franca S. Angeli • Nicolas Amabile • Mia Shapiro • Rachel Mirsky • Lauren Bartlett • \\ Yan Zhang • Renu Virmani • Kanu Chatterjee • Andrew Boyle • William Grossman • \\ Yerem Yeghiazarians
}

Published online: 1 September 2010

(C) The Author(s) 2010. This article is published with open access at Springerlink.com

\begin{abstract}
Purpose Erythropoietin (EPO) and granulocyte colony stimulating factor (GCSF) have generated interest as novel therapies after myocardial infarction (MI), but the effect of combination therapy has not been studied in the large animal model. We investigated the impact of prolonged combination therapy with EPO and GCSF on cardiac function, infarct size, and vascular density after MI in a porcine model.

Methods MI was induced in pigs by a 90 min balloon occlusion of the left anterior descending coronary artery. 16 animals were treated with EPO+GCSF, or saline (control group). Cardiac function was assessed by echocardiography and pressure-volume measurements at baseline, 1 and 6 weeks post-MI. Histopathology was performed 6 weeks post-MI.

Results At week 6, EPO+GCSF therapy stabilized left ventricular ejection fraction, $(41 \pm 1 \%$ vs. $33 \pm 1 \%, p<0.01)$ and improved diastolic function compared to the control group. Histopathology revealed increased areas of viable myocardium and vascular density in the $\mathrm{EPO}+\mathrm{GCSF}$ therapy, compared to the control. Despite these encouraging results, in a historical analysis comparing combination
\end{abstract}

F. S. Angeli $\cdot$ N. Amabile $\cdot$ M. Shapiro $\cdot$ R. Mirsky $\cdot$ Y. Zhang $\cdot$

K. Chatterjee $\cdot$ A. Boyle $\cdot$ W. Grossman $\cdot$ Y. Yeghiazarians $(\triangle)$

Division of Cardiology, Department of Medicine,

University of California,

505 Parnassus Avenue, L-523, Box 0103,

San Francisco, CA 94143-0103, USA

e-mail: yeghiaza@medicine.ucsf.edu

L. Bartlett $\cdot$ R. Virmani

CVpath Institute,

19 Firstfield Road,

Gaithersburg, MD, USA therapy with monotherapy with EPO or GCSF, there were no significant additive benefits in the LVEF and volumes overtime using the combination therapy.

Conclusion Our findings indicate that $\mathrm{EPO}+\mathrm{GCSF}$ combination therapy promotes stabilization of cardiac function after acute MI. However, combination therapy does not seem to be superior to monotherapy with either EPO or GCSF.

Key words Erythropoietin - Granulocyte colony stimulating factor $\cdot$ Myocardial infarction $\cdot$ Cardiac remodeling $\cdot$ Cytokine

\section{Introduction}

Myocardial infarction (MI) is a leading cause of morbidity and mortality in Western countries [1]. Despite advances in the management of MI, the number of patients with congestive heart failure continues to grow and remains associated with increased risk of death [1]. Novel therapeutic approaches targeted to repairing myocardial damage have been the focus of intense research over the recent years [2-5].

Recently, granulocyte colony stimulating factor (GCSF) and Erythropoietin (EPO) have emerged as promising candidates for treatment of acute ischemic heart disease. Despite promising pre-clinical data using GCSF [6, 7], human clinical trials in acute MI patients, while generally reassuring in terms of safety, have been disappointing from the standpoint of clinical benefit, raising questions about the adequacy of GCSF monotherapy. Nevertheless, we have recently shown that GCSF therapy mobilizes bone marrow cells, enhances neovascularization, and prevents further 
deterioration of LV function in a porcine model of MI with lower LVEF [8]. In line with our results, a recent metaanalysis suggests that GCSF may be potentially beneficial in patients with larger infarcts who have a lower LVEF $(<50 \%)[9]$.

EPO has been shown to improve myocardial contractility [10], reduce cellular damage and apoptosis [11], and increase neovascularization, leading to reduced infarct size and improved cardiac function in rodent models of MI [1214]. For the first time, our group has recently shown that in a large animal MI model, prolonged therapy (4 weeks) with EPO decreases infarct size, mobilizes bone marrow cells, enhances neovascularization and results in improvements in ventricular remodeling and function in a porcine model of acute MI [15].

Given the diversity of cytokines and their overlapping functions [16-18] and the beneficial effects of EPO and GCSF therapy post MI, we hypothesized that combination therapy with EPO and GCSF would enhance angiogenesis, and decrease infarct size and, therefore, would result in concomitant improvements in ventricular remodeling and function in a porcine model of acute MI with reperfusion. The current manuscript builds on the previous work and examines whether the combination of EPO and GCSF would be safe and effective in improving the cardiac function post-MI. To our knowledge, no prior study has examined the effect of $\mathrm{EPO}+\mathrm{GCSF}$ combination therapy after MI in the large animal model. Of note, since limited funding was secured for completion of this combination cytokine study, the current results were compared to historical and previously published EPO and GCSF monotherapy arms $[8,15]$ by our group. To keep this comparison appropriate, all aspects of the study amongst the groups were performed similarly and by the same operators.

\section{Methods}

Animals

This study was carried out in accordance with the guidelines of the National Institutes of Health with a protocol approved by the Institutional Animal Care and Use Committee of University of California San Francisco (UCSF). Eighteen Yorkshire-Landrace pigs weighing 35$43 \mathrm{~kg}$ were obtained (Pork Power, Turlock, CA) for this study.

\section{Induction of MI}

MI was induced by a 90 min balloon occlusion of the mid left anterior descending coronary artery (LAD) as previously published by our group $[8,15,19]$. Briefly, general anesthesia was induced by intramuscular injection of ketamine $(20 \mathrm{mg} / \mathrm{kg})$, xylazine $(2 \mathrm{mg} / \mathrm{kg})$ and atropine $(0.04 \mathrm{mg} / \mathrm{kg})$ then maintained with $2 \%$ isoflurane. The levels of anesthesia were kept the same at all study time points. Continuous blood pressure, oxygen saturation, and telemetry monitoring were performed during all procedures. A $6 \mathrm{~F}$ sheath was placed in the femoral artery and after systemic heparinization (100-200 U/kg), the coronary artery was selectively engaged with a $6 \mathrm{~F}$ HS0.75 guide catheter. A standard guide wire was placed in the $\mathrm{LAD}$, and a 2.5 to $3.5 \times$ $15 \mathrm{~mm}$ coronary angioplasty balloon delivered to the mid LAD just distal to the second diagonal branch. Balloon inflation ( $\sim \mathrm{atm})$ for $90 \mathrm{~min}$ was performed to induce MI. Complete occlusion with balloon inflation and LAD patency after balloon deflation was confirmed angiographically. Intravenous (IV) amiodarone (75 $\mathrm{mg}$ over $10 \mathrm{~min}$ ) and lidocaine ( $1 \mathrm{mg} / \mathrm{kg}$ IV bolus, followed by $1 \mathrm{mg} / \mathrm{min}$ infusion) were started prior to balloon occlusion, with additional lidocaine (1-3 mg/kg IV bolus) given at the discretion of the operator for significant ventricular arrhythmias. Animals were medicated with atenolol ( $25 \mathrm{mg}$ orally) daily starting 3 days post-MI. Prior to sacrifice, all animals underwent repeat coronary angiography.

\section{Treatment protocol}

Animals were assigned to one of two treatment groups using the same protocol previously published by our group testing EPO and GCSF monotherapy [8, 15]: 1) Long-acting EPO analog (Aranesp, Amgen, Thousand Oaks, CA) was given as IV bolus at the time of reperfusion $(0.9 \mathrm{ug} / \mathrm{Kg})$, then as weekly SC injections for 4 weeks $(0.4 \mathrm{ug} / \mathrm{Kg})$, and GCSF (Neupogen, Amgen, Thousand Oaks, CA) was given as IV bolus at time of reperfusion and then daily SC injections from day 5 to 9 post MI; and 2) control group (normal saline in equivalent volume given IV and $\mathrm{SC}$ to match the administration of the EPO therapy group). EPO dosages were selected following clinical data showing safety and feasibility with a single bolus of a fixed dose of darbepoetin [20], clinical studies addressing safety of chronic use of darbepoetin in patients with chronic renal failure [21], and pre-clinical data showing improvement in cardiac function and neovascularization using prolonged EPO therapy in a dose that did not increase the hematocrit [14]. GCSF dosages were selected following clinical data showing safety and feasibility of similar dose post MI [9].

Animal monitoring

Behavior, excreta, attitude (alertness, responsiveness, appetite), signs of respiratory distress (respiratory rate, respiratory pattern), motor weakness, and swelling extremities were monitored daily for the length of study. Arterial blood 
pressure was monitored at baseline, immediately post reperfusion, one, and 6 weeks post MI.

Blood sampling and laboratory analysis

Whole blood samples were collected at baseline (prior to $\mathrm{MI}$ ), at $2 \mathrm{~h}$, on week one to four after MI induction, and then at sacrifice. Hemoglobin, hematocrit, white blood count, creatinine levels, creatine kinase MB fraction (CK$\mathrm{MB})$, and troponin I (TnI) were measured by the animal core laboratory (IDEEX, Sacramento, CA).

\section{Echocardiography}

Transthoracic echocardiography (TTE) was performed at baseline, 1 and 6 weeks after MI using an Acuson 128XP machine with $\mathrm{S} 3(1-3 \mathrm{MHz})$ and $\mathrm{S} 8(3-8 \mathrm{MHz})$ probes (Siemens, Malvern, PA) as previously published by our group [8, 15, 19]. Long- and short-axis parasternal views and 4- and 2- chamber apical views were acquired. Left ventricular end-diastolic volume (LVEDV), end-systolic volume (LVESV), and ejection fraction (LVEF) were measured using the area/length method. The wall motion index (WMI) was calculated, using the method previously described by the American Society of Echocardiography [22], by grading the standard 17 myocardial segments (normal=1, hypokinesis (reduced endocardial motion and wall thickening in systole) $=2$, akinesis (absence of inward endocardial motion or wall thickening in systole) $=3$, dyskinesis (outward motion or "bulging" of the segment in systole, usually associated with thin, scarred myocardium) $=4$, aneurysm=5), and dividing the sum of the scores by the number of segments visualized. For all above parameters, at least three loops per scan were selected and the results presented as an average of the readings. Readings were made by blinded operators. The interobserver variability (made from different readings of recorded loops) expressed using coefficient of variation in the measurement of LVEDV and LVESV was $4.3+/-5.7 \mathrm{~mL}$ and $1.4+/-2.9 \mathrm{~mL}$, respectively, corresponding to variability in absolute LVEF of $2+/-3 \%$. The intraobserver variability for WMSI (mean difference between measures 1 and 2) was $1.9 \%$, while the interobserver variability (mean difference between observers 1 and 2) was $2.5 \%$.

Left ventricular pressure-volume (PV) data were collected at baseline, 1 week, and 6 weeks after MI as previously published by our group [8, 15, 19]. Conductance and pressure signals were acquired using a dual field $5 \mathrm{~F} 12$ electrode pigtail PV catheter (Millar Instruments, Houston, TX) connected to a Leycom CFL-512 console (CD Leycom, Zoetermeer, Netherlands) via a TC-510 (Millar) pressure control unit and a patient module (CD Leycom) as previously described [19].
Pressure volume measurements

The ventricular end-systolic and end-diastolic pressurevolume relationships are considered as gold standards in the characterization of intrinsic ventricular pump properties [23-25]. In this study, the PV catheter was inserted in the long axis of the left ventricle and oriented with segment one in the apex and segment seven in the aortic outflow as previously reported [8, 15, 19]. Inferior vena cava (IVC) occlusion was performed with a 7F, $34 \mathrm{~mm}$ Amplatzer sizing balloon (AGA Medical, Plymouth, MN) introduced via the femoral vein, inflated for $6-10 \mathrm{~s}$ to achieve $\sim 50 \%$ drop in arterial blood pressure. Continuous data were acquired at a sampling frequency of $250 \mathrm{~Hz}$ during the steady state and IVC occlusion.

PV data were analyzed offline using the Conduct NT software (CD Leycom) by a blinded operator with a $10 \mathrm{~Hz}$ filter as previously described [19, 26]. Briefly, data were
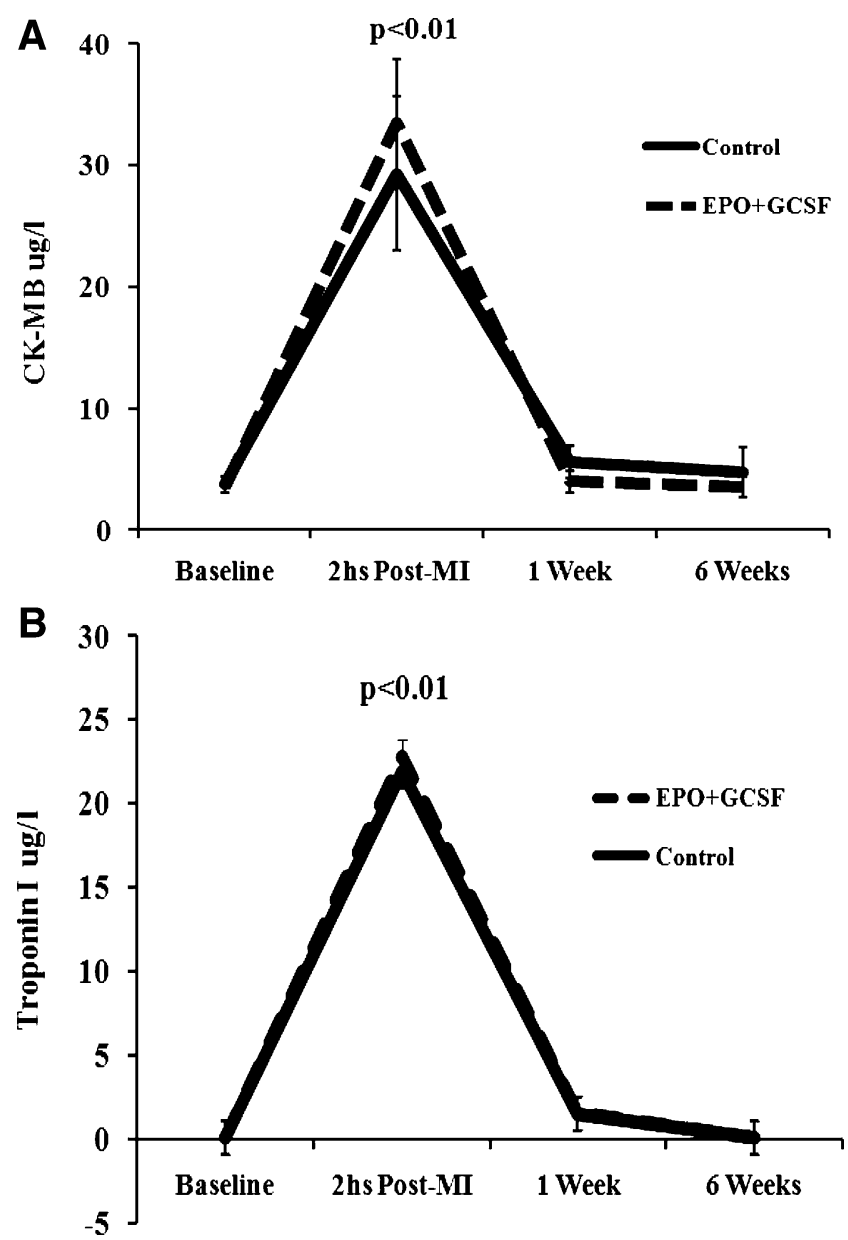

Fig. 1 Enzymatic curve for CK-MB and Troponin I. a CK-MB (ug/ml); each line represents the mean of one experimental group. b Troponin I (ug.ml); each line represents the mean of one experimental group. Both enzymes were significantly increased $2 \mathrm{~h}$ post MI, returning to baseline at 6 weeks. There were no differences between EPO+GCSF and Control in each one of the time-points 


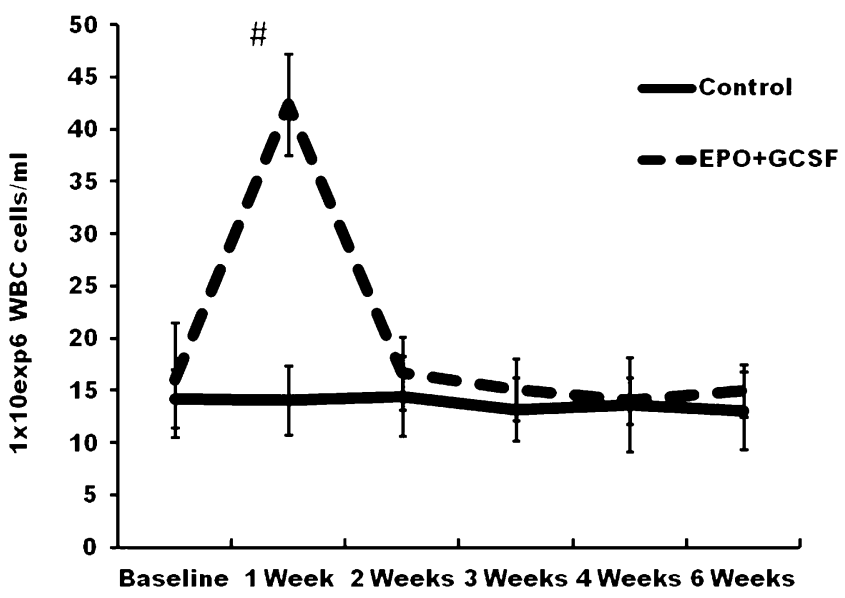

Fig. 2 Leukocyte response to cytokine therapy. EPO+GCSF combination therapy induced a significant increase in WBC 1 week after MI compared to baseline and Control group (both, $\# P<0.01$ )

calibrated for parallel conductance $\left(\mathrm{V}_{\mathrm{c}}\right)$ and alpha $(\alpha)$ based on volumes derived from transthoracic echocardiographic images collected at the beginning of each case. Using conductance data, and the $\alpha$ and $\mathrm{V}_{\mathrm{c}}$ calculations, the Conduct NT software calculated ventricular volumes as previously described [27]. Steady state data included heart rate (HR), maximum rate of pressure change in systole $\left(\mathrm{dP} / \mathrm{dt}_{\max }\right)$, decline with relaxation $\left(\mathrm{dP} / \mathrm{dt}_{\min }\right)$, left ventricular enddiastolic pressure (LVEDP), end-systolic pressure (LVESP), LVESV, and LVEDV. Stroke volume (SV) was recorded as LVEDV-LVESV, cardiac output $(\mathrm{CO})$ as SV $\times$ HR, LVEF as SV/LVEDV, and stroke work (SW) as the area enclosed by the PV loop.

Diastolic function was evaluated during steady state by the time constant of isovolumic relaxation, $\tau$, and the $\mathrm{dP} / \mathrm{dt}_{\text {min }}$. $\tau$ was computed as described by Raff and Glantz using pressure recorded during the isovolumetric relaxation period which is the period from the time of $\mathrm{dP} / \mathrm{dtmin}$ to the time when left ventricular pressure falls to $5 \mathrm{mmHg}$ above the end-diastolic pressure of the following beat [28]. $\tau$ was calculated as the negative inverse of the linear slope of $\mathrm{dp} / \mathrm{dt}$ vs. pressure during this period.

Data obtained during IVC occlusion were used to calculate the linear end-systolic pressure-volume relation (characterized by the slope; also called end-systolic elastance $\left(\mathrm{E}_{\mathrm{es}}\right)$ and an intercept, $\mathrm{V}_{0}$, and the preload recruitable stroke work (PRSW, or slope of SW versus LVEDV curve) [29].
Table 1 Echocardiographic parameters over time

The values are expressed as the mean \pm standard error (in parentheses)

$L V E F$ left ventricle ejection fraction, LVEDV LV end-diastolic volume, LVESV LV end-systolic volume, $W M I$ wall motion index, $H R$ heart rate

NS Non significant

${ }^{\text {a }}$ Significance of differences "between groups" was tested by an unpaired $t$ test

${ }^{\mathrm{b}}$ Change from baseline value; significance of post-hoc test in repeated measures ANOVA design ${ }^{*} p<0.05$ vs. baseline, ${ }^{* *} p<0.01$ vs. baseline, ${ }^{* * *} p<0.05$ vs. 1 week, ${ }^{* * * * *} p<0.01$ vs. 1 week

\begin{tabular}{|c|c|c|c|}
\hline Parameter & Control $(n=8)$ & $\mathrm{EPO} \pm \mathrm{GCSF}(n=8)$ & $t$ Test $^{\mathrm{a}}$ \\
\hline \multicolumn{4}{|l|}{ LVEF (\%) } \\
\hline Baseline & $55.2(1.2)$ & $56.8(1.3)$ & NS \\
\hline 1 Week post $\mathrm{MI}^{\mathrm{b}}$ & $41.3(2.1)^{* *}$ & $41.1(1.6)^{* *}$ & NS \\
\hline 6 Weeks post $\mathrm{MI}^{\mathrm{b}}$ & $33.2(1.5)^{* *}, * * *$ & $41(1.2)^{* *}$ & $p<0.01$ \\
\hline Repeated measures ANOVA (main effect) & $p<0.01$ & $p<0.01$ & \\
\hline \multicolumn{4}{|l|}{ LVEDV (mL) } \\
\hline Baseline & $52.2(1.9)$ & $53.2(0.9)$ & NS \\
\hline 1 Week post $\mathrm{MI}^{\mathrm{b}}$ & $63.5(2)^{* *}$ & $62.5(1.5)^{* *}$ & NS \\
\hline 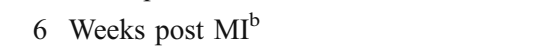 & $73.5(2.5)^{* *}, * * * *$ & $67.9(2)^{* *}, * * * *, * * * *$ & $p=0.04$ \\
\hline Repeated measures ANOVA (main effect) & $p<0.01$ & $p<0.01$ & \\
\hline \multicolumn{4}{|l|}{ LVESV (mL) } \\
\hline Baseline & $23.6(1.2)$ & $22.9(0.9)$ & NS \\
\hline 1 Week post $\mathrm{MI}^{\mathrm{b}}$ & $37.2(1.8)^{* *}$ & $36.8(1.2)^{* *}$ & NS \\
\hline 6 Weeks post $\mathrm{MI}^{\mathrm{b}}$ & $49.5(1.9)^{* *}, * * * *$ & $40.7(1.5)^{* *}$ & $p=0.01$ \\
\hline Repeated measures ANOVA (main effect) & $p<0.01$ & $p<0.01$ & \\
\hline \multicolumn{4}{|l|}{ WMI } \\
\hline Baseline & 1 & 1 & \\
\hline 1 Week post $\mathrm{MI}^{\mathrm{b}}$ & $1.7(0.1)^{* *}$ & $1.6(1)^{* *}$ & NS \\
\hline 6 Weeks post $\mathrm{MI}^{\mathrm{b}}$ & $1.9(0.1)^{* *}, * * * *$ & $1.6(1)^{* *}$ & $p<0.01$ \\
\hline Repeated measures ANOVA (main effect) & $p<0.01$ & $p<0.001$ & \\
\hline \multicolumn{4}{|l|}{ HR (bpm) } \\
\hline Baseline & $79(4)$ & $84(7)$ & NS \\
\hline 1 Week post $\mathrm{MI}^{\mathrm{b}}$ & $84(6)$ & $87(4)$ & NS \\
\hline 6 Weeks post $\mathrm{MI}^{\mathrm{b}}$ & $87(6)$ & $83(6)$ & NS \\
\hline Repeated measures ANOVA (main effect) & NS & NS & \\
\hline
\end{tabular}


A
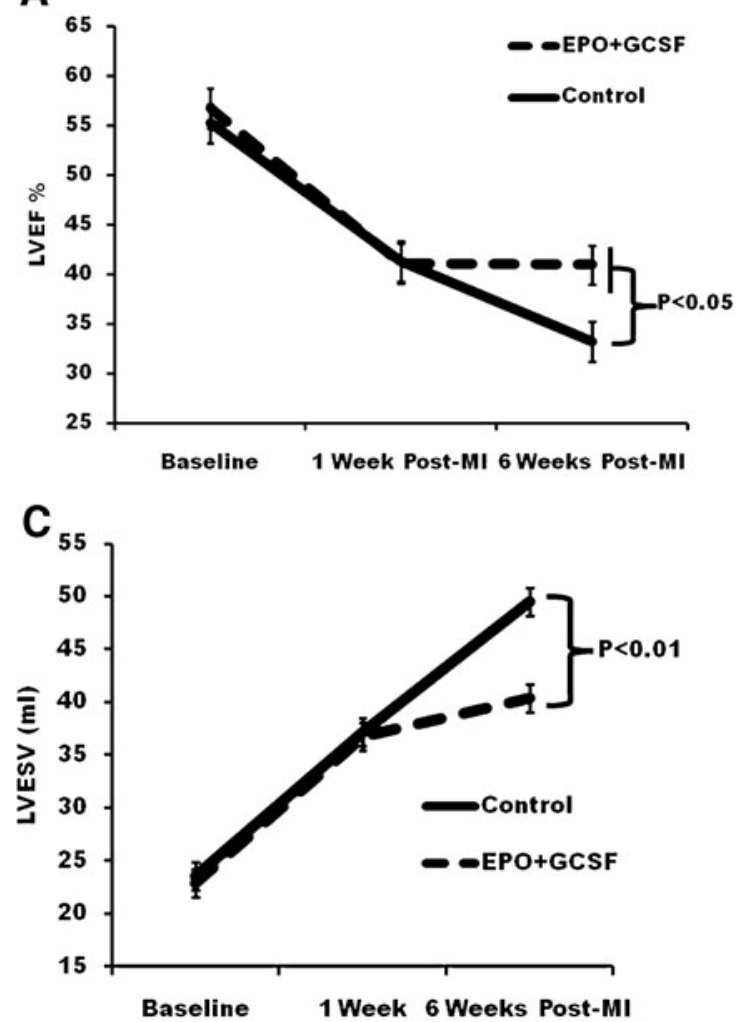

Fig. 3 Changes in LV function and volumes over time following myocardial infarction by echocardiography. a Left ventricular ejection fraction $(L V E F)$; each line represents the mean of one experimental group. LVEF continue to decrease in controls, while combination
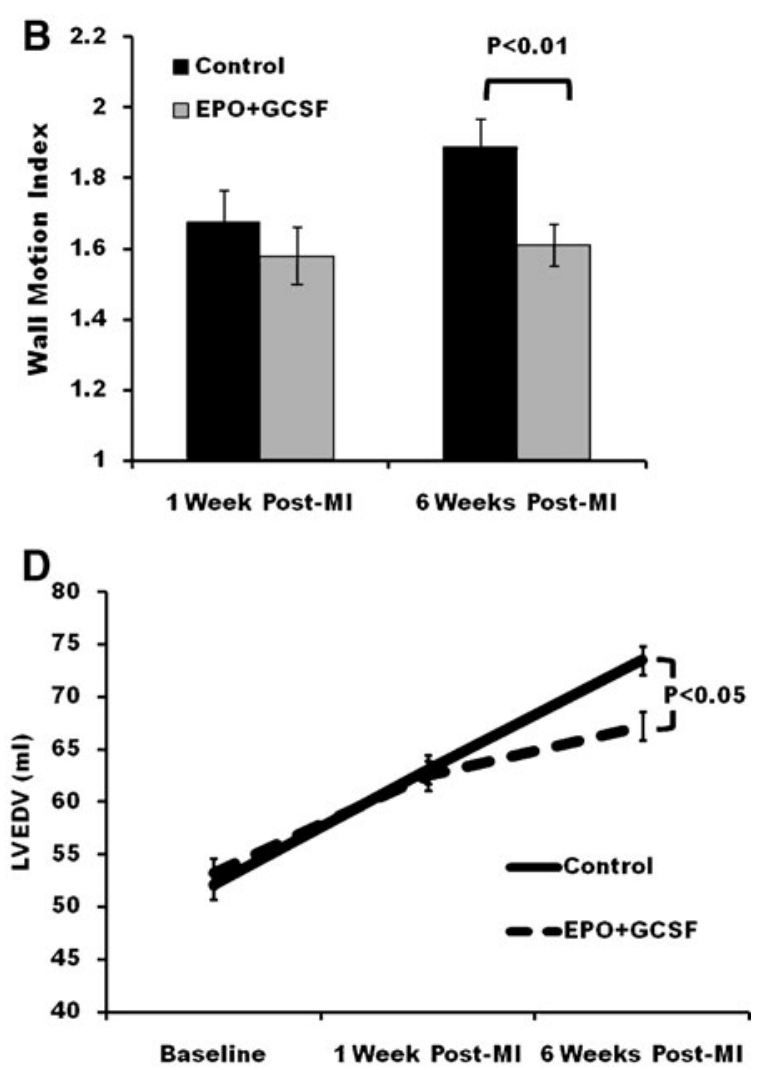

therapy stabilizes LVEF. b Wall motion index does not differ at 1 week, but is better on the EPO+GCSF therapy compared to control at 6 weeks $\mathbf{c}$ End systolic volume and $\mathbf{d}$ end diastolic volume at 6 weeks post-MI. Data are shown as mean \pm SEM
Histological and immunohistochemistry analysis

All animals were sacrificed 6 weeks after MI and their hearts excised, weighed, and any gross surface abnormalities recorded. Histological and immunohistochemistry was performed in a blinded manner by CV Path Institute, Inc, MD. The ventricles were serially sliced at approximately $1 \mathrm{~cm}$ intervals parallel to the posterior atrioventricular sulcus from the apex to the base as previously describe by our group [8, 15, 19]. The thickness of each slice was measured and recorded. Digital images were taken for morphometric analysis of LV area, infarct size, and thickness, using IPLab software (Scanalytics, Rockville, MD). Infarct size was defined as a thinned and pale region of the anterior LV wall [30] and did not account for areas of viable tissue. Myocardial tissue for paraffin embedding was taken from the basal, mid, and apical-cavity levels. The three levels were then divided clockwise, beginning with the interventricular groove, into sixteen segments as described before [31]. Myocardial sections were transferred to $15 \%$ sucrose, dehydrated in a graded series of alcohols, and embedded in paraffin. Sections $(4-5 \mu \mathrm{m})$ were mounted on charged slides and stained with hematoxylin and eosin,
Masson's Trichrome to evaluate for fibrosis using the IPLab software. The fibrotic areas from the 16 ventricle segments were summed and presented as a percent of the total LV area. The infarct zone (IZ) was defined as the arc of the left ventricle containing scar tissue.

For vessel density, myocardial tissue for paraffin embedding was taken from the basal, mid, and apical levels, and sectioned circumferentially into 4 adjacent transmural areas to include the central area of infarction, 2 adjacent border areas (border zone for capillary and arteriole measurements were defined at sites $1.2 \mathrm{~mm}$ and $2.0 \mathrm{~mm}$ outside the zone of infarction), and a control noninfarcted region (remote zone).

Immunohistochemical staining with a biotinylated lectin antibody (Dolichos biflorus, DBA, Sigma, St. Louis, MO) was used for the identification of capillaries. A monoclonal antibody against smooth muscle actin clone 1A4 (dilution $1: 2000$, Sigma) was used for the identification of vascular smooth muscle cells for identification of arteries and arterioles. Vascular density was measured at mid-ventricle region, in six to nine high power fields per section. Capillary (200X magnification), artery and arteriolar $(100 \mathrm{X})$ density were measured and expressed as the mean 
Table 2 Conductance catheter measurements over time

\begin{tabular}{|c|c|c|c|}
\hline Parameter & Control $(n=8)$ & $\mathrm{EPO} \pm \mathrm{GCSF}(n=8)$ & $t$ Test $^{\mathrm{a}}$ \\
\hline \multicolumn{4}{|l|}{ HR (bpm) } \\
\hline Baseline & $80(3)$ & $86(5)$ & NS \\
\hline 1 Week post $\mathrm{MI}^{\mathrm{b}}$ & $83(3)$ & $85(4)$ & NS \\
\hline 6 Weeks post $\mathrm{MI}^{\mathrm{b}}$ & $88(5)$ & $92(3)$ & NS \\
\hline Repeated measures ANOVA (main effect) & NS & NS & \\
\hline \multicolumn{4}{|l|}{ MAP(mmHg) } \\
\hline Baseline & $82(4)$ & $85(3)$ & NS \\
\hline 1 Week post $\mathrm{MI}^{\mathrm{b}}$ & $80(6)$ & $88(4)$ & NS \\
\hline 6 Weeks post $\mathrm{MI}^{\mathrm{b}}$ & $86(8)$ & $85(5)$ & NS \\
\hline Repeated measures ANOVA (main effect) & NS & NS & \\
\hline \multicolumn{4}{|l|}{ LVESP $(\mathrm{mmHg})$} \\
\hline Baseline & $86(2)$ & $89(5)$ & NS \\
\hline 1 Week post $\mathrm{MI}^{\mathrm{b}}$ & $90(4)$ & $93(3)$ & NS \\
\hline 6 Weeks post $\mathrm{MI}^{\mathrm{b}}$ & $76(3)^{*}, * * * *$ & $84(4)$ & NS \\
\hline Repeated measures ANOVA (main effect) & $p<0.02$ & NS & \\
\hline \multicolumn{4}{|l|}{ LVEDP (mmHg) } \\
\hline Baseline & $3.1(0.3)$ & $2.9(0.5)$ & NS \\
\hline 1 Week post $\mathrm{MI}^{\mathrm{b}}$ & $9.9(1.4)^{* *}$ & $8.8(1.1)^{* *}$ & NS \\
\hline 6 Weeks post $\mathrm{MI}^{\mathrm{b}}$ & $6(0.5)^{*, * * * *}$ & $4.3(1.1)^{* * * *}$ & NS \\
\hline Repeated measures ANOVA (main effect) & $p<0.01$ & $p<0.01$ & \\
\hline \multicolumn{4}{|l|}{$\mathrm{SV}(\mathrm{mL})$} \\
\hline Baseline & $28.6(1)$ & $30.4(0.7)$ & NS \\
\hline 1 Week post $\mathrm{MI}^{\mathrm{b}}$ & $26.3(1.5)$ & $25.7(1.3)^{*}$ & NS \\
\hline 6 Weeks post $\mathrm{MI}^{\mathrm{b}}$ & $21.4(0.7)^{* *}, * * * *$ & $27.7(1.4)$ & $p<0.01$ \\
\hline Repeated measures ANOVA (main effect) & $p<0.01$ & $p=0.03$ & \\
\hline \multicolumn{4}{|l|}{ Ees $(\mathrm{mmHg} / \mathrm{ml})$} \\
\hline Baseline & $1.7(0.2)$ & $1.4(0.2)$ & NS \\
\hline 1 Week post $\mathrm{MI}^{\mathrm{b}}$ & $1.2(0.3)$ & $1.8(0.3)$ & NS \\
\hline 6 Weeks post $\mathrm{MI}^{\mathrm{b}}$ & $1.6(0.3)$ & $1.7(0.2)$ & NS \\
\hline Repeated measures ANOVA (main effect) & NS & NS & \\
\hline \multicolumn{4}{|l|}{ Vo intercept (ml) } \\
\hline Baseline & $-37.2(7)$ & $-42.9(10)$ & NS \\
\hline 1 Week post $\mathrm{MI}^{\mathrm{b}}$ & $-25.9(6)$ & $-28(5)$ & NS \\
\hline 6 Weeks post $\mathrm{MI}^{\mathrm{b}}$ & $11.4(5)^{* *}, * * * *$ & $-11(6)^{* *}$ & $p<0.01$ \\
\hline Repeated measures ANOVA (main effect) & $p<0.01$ & $p<0.01$ & \\
\hline \multicolumn{4}{|l|}{ PRSW (mmHg) } \\
\hline Baseline & $53(3)$ & $51(1)$ & NS \\
\hline 1 Week post $\mathrm{MI}^{\mathrm{b}}$ & $41(2)^{* *}$ & $40(2)^{* *}$ & NS \\
\hline 6 Weeks post $\mathrm{MI}^{\mathrm{b}}$ & $34(1)^{* *}, * * *$ & $41(2)^{* *}$ & $p<0.05$ \\
\hline Repeated measures ANOVA (main effect) & $p<0.01$ & $p<0.01$ & \\
\hline \multicolumn{4}{|l|}{$\mathrm{dP} / \mathrm{dtmax}(\mathrm{mmHg} / \mathrm{s})$} \\
\hline Baseline & $1270(65)$ & $1255(42)$ & NS \\
\hline 1 Week post $\mathrm{MI}^{\mathrm{b}}$ & $1035(53)^{* *}$ & $1020(36)^{* *}$ & NS \\
\hline 6 Weeks post $\mathrm{MI}^{\mathrm{b}}$ & $842(38)^{* *}, * * * *$ & $1038(20)^{* *}$ & $p<0.01$ \\
\hline Repeated measures ANOVA (main effect) & $p<0.01$ & $p<0.01$ & \\
\hline \multicolumn{4}{|l|}{$\mathrm{dP} / \mathrm{dtmin}(\mathrm{mmHg} / \mathrm{s})$} \\
\hline Baseline & $-1156(18)$ & $-1168(20)$ & NS \\
\hline 1 Week post $\mathrm{MI}^{\mathrm{b}}$ & $-1024(37)^{* *}$ & $-1068(23)^{* *}$ & NS \\
\hline 6 Weeks post $\mathrm{MI}^{\mathrm{b}}$ & $-890(30)^{* *}, * * * *$ & $-1035(21)^{* *}$ & $p<0.01$ \\
\hline
\end{tabular}


Table 2 (continued)

\begin{tabular}{llll}
\hline Parameter & Control $(n=8)$ & EPO \pm GCSF $(n=8)$ & $t$ Test ${ }^{\mathrm{a}}$ \\
\hline $\begin{array}{l}\text { Repeated measures ANOVA (main effect) } \\
\begin{array}{l}(\mathrm{ms}) \\
\text { Baseline }\end{array}\end{array}$ & $p<0.01$ & $p<0.01$ \\
1 Week post $\mathrm{MI}^{\mathrm{b}}$ & $57(1)$ & $56(1)$ & $\mathrm{NS}$ \\
6 Weeks post MI & $69(4)^{* *}$ & $64(2)^{* *}$ & $\mathrm{NS}$ \\
Repeated measures ANOVA (main effect) & $62(1)^{*}, * * *$ & $56(1)^{* * * * *} \quad p<0.01$ \\
\hline
\end{tabular}

The values are expressed as the mean \pm by standard error (in parentheses)

$H R$ heart rate, MAP mean arterial pressure, LVESP left ventricular end-systolic pressure, LVEDP left ventricular end-diastolic pressure, $S V$ stroke volume, Ees linear end-systolic pressure-volume relation or end-systolic elastance, $V_{0}$ Volume zero Ees intercept, $P R S W$ preload-recruitable stroke work, $d P / d t_{\max }$ maximum rate of change of left ventricular pressure with time, $d P / d t_{\min }$ peak of pressure decay, $\tau$ time constant of isovolumic relaxation, $M I$ myocardial infarction

NS Non significant

a Significance of differences "between groups" was tested by an unpaired $t$ test

${ }^{\mathrm{b}}$ Change from baseline value; significance of post-hoc test in repeated measures ANOVA design ${ }^{*} p<0.05$ vs. baseline, ${ }^{* *} p<0.01$ vs. baseline, $* * * p<0.05$ vs. 1 week, $* * * *<0.01$ vs. 1 week

$\left( \pm\right.$ SEM) number of vessels per $\mathrm{mm}^{2}$. The measured total tissue area was corrected for remaining interstitial space.

\section{Statistical analysis}

All results are expressed as means \pm standard error of the mean (SEM). A repeated measures ANOVA model (SigmaStat 3.5, Systat Software, San Jose, CA) was used to test the responses of examined parameters (measured at baseline, week 1 and 6) in the experimental variants (Control, EPO+GCSF). The within-subject design included an overall $F$ test of the main effects and then a post-hoc pairwise comparison of the values measured at 1 week and 6 weeks against the baseline, and 6 weeks against 1 week, using the Holm-Sidak method. Significance of differences between groups (Control, EPO+GCSF) was tested by an unpaired $t$ test. A historical analysis using repeated measures ANOVA model (SigmaStat 3.5, Systat Software, San Jose, CA) was used to test the responses of examined parameters (measured at baseline, week1 and 6) in all experimental variants (EPO+GCSF). Correlations were performed using the Person method. A $p<0.05$ was considered statistically significant in all the employed tests.

\section{Results}

Two animals died during creation of the MI model, leaving sixteen animals for the study. Patency of the coronaries was confirmed by angiography post reperfusion in all remaining animals. Mean arterial pressure immediately after reperfusion remained stable compared to baseline and did not differ between groups (mean $83 \pm 4$ at baseline vs. $81 \pm 4$ after reperfusion, $p=\mathrm{NS}$ ). No adverse clinical events related to the drugs were noted during the study, including thromboembolic events and hypertension. CRP levels remained stable over time in both groups. As shown on Fig. 1, CK-MB and TnI levels did not differ between groups and, as expected, were significantly elevated two hours after the induction of the MI, returning to baseline thereafter (mean CK-MB $3.8 \pm 1.9$ to $29.2 \pm 7.1 \mu \mathrm{g} / \mathrm{L}, p<0.01$; and mean TnI $0.48 \pm 0.07$ to $22.5 \pm 2.3 \mu \mathrm{g} / \mathrm{L}, p<0.01)$. All animals underwent repeat coronary angiography prior to sacrifice at the end of the study and LAD patency was documented. No differences in collateral circulation compared to baseline angiography or amongst the groups were present.

Cytokine therapy mobilizes bone marrow cells

EPO+GCSF group had significantly increased WBC counts at 1 week post-MI (Fig. 2). The mononuclear fraction count (lymphocytes plus monocytes) were also significantly higher at 1 week post-MI on the EPO+GCSF group compared to baseline $(p<0.01)$ and to Control (respectively, $12.6 \pm 0.5$ vs. $8.7 \pm 0.710^{6}$ cells $\left./ \mathrm{ml}\right), p<0.01$ At week 4 post-MI, EPO + GCSF therapy induced a significant increase in the hemoglobin levels compared to baseline (respectively, $10.8 \pm 0.4$ to $13.8 \pm 0.6 \mathrm{~g} / \mathrm{dl} ; p<0.01)$.

\section{Echocardiographic parameters: EPO+GCSF preserves} LVEF and prevents LV dilation over time

Echocardiographic parameters are shown in Table 1. At 6 weeks post-MI, EPO+GCSF group stabilized LVEF, while the control group demonstrated a statistically significant further deterioration of function ( $0 \pm 1$ vs. $-7 \pm 1 \%, p<0.01$ vs. control) compared to week 1 post MI (Fig. 3a). The wall 


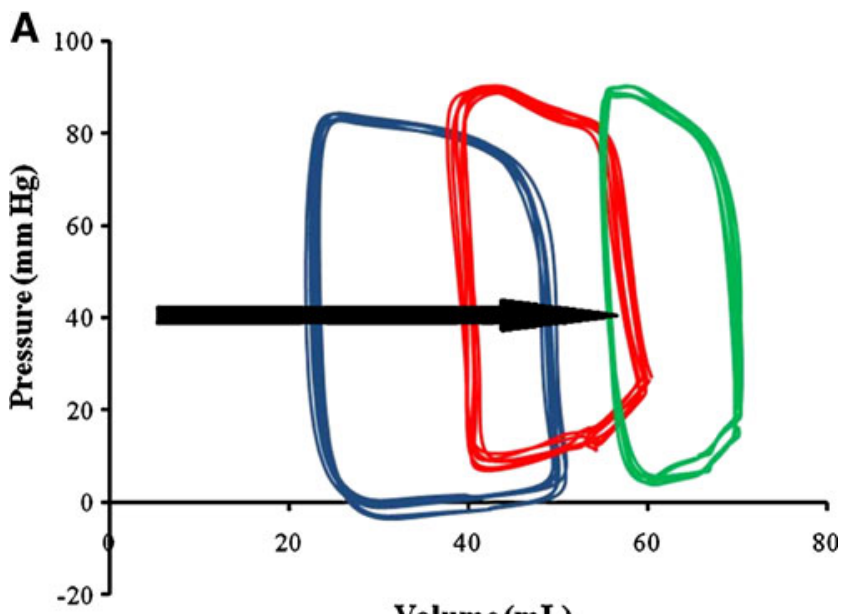

Volume (mL)

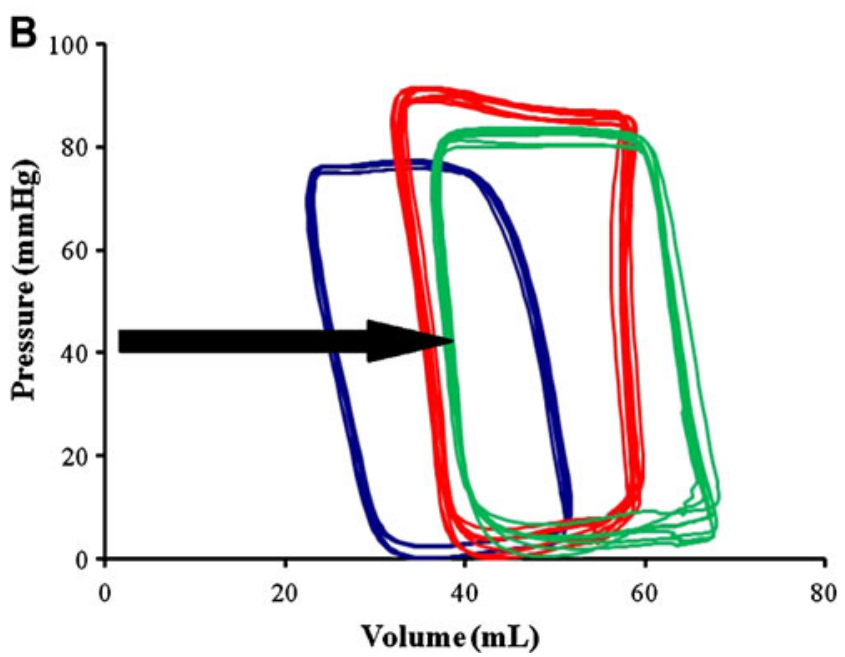

Fig. 4 EPO + GCSF preserves hemodynamics over time. Representative steady-state $\mathrm{PV}$ loops from one animal at baseline (blue), 1 week post-MI (red) and 6 weeks post-MI (green) from Control a and EPO+GCSF combination therapy b. After infarction, the PV loops narrowing is more evident in the Control animals compare to the EPO+GCSF group, indicating reduction in stroke work, and shift rightward due to increasing volumes (black arrows)

motion score was better in the EPO+GCSF group compared to the control (Fig. 3b), corroborating the LVEF findings. LVESV and LVEDV were also lower in the EPO+GCSF group at 6 weeks compared to the control (Fig. 3c and d).

PV-loop parameters: Cytokine therapy prevents further impairment of systolic function after AMI

The hemodynamic parameters are summarized in Table 2 . At 6 weeks, there was a significant increase in SV in the EPO+GCSF group compared to control $(27.7 \pm 1.4$ vs. $21.4 \pm 0.7, p<0.01$ ) (Fig. 4). Moreover, the peak positive $\mathrm{dP} / \mathrm{dt}$ in the $\mathrm{EPO}+\mathrm{GCSF}$ group was higher than the control group. The linear Ees was unchanged overtime and did not differ between groups, but there was a significant difference in the rightward movement of the $\mathrm{V}_{0}$, indicating increased heart size in the control group in comparison to EPO+GCSF therapy (Fig. 4). As previously reported by us and others $[19,32,33]$, the non-significant changes in the slope of the Ees could be a result of changes in loading conditions, LV dimensions, and regional morphological changes which are difficult to evaluate with the conductance catheter method over time but these reflect what we expect to see in clinical settings. On the other hand, the slope of the PRSW is a reasonable linear, afterloadindependent relationship, and a well-described contractility index in intact animal models [29]. In our study, the
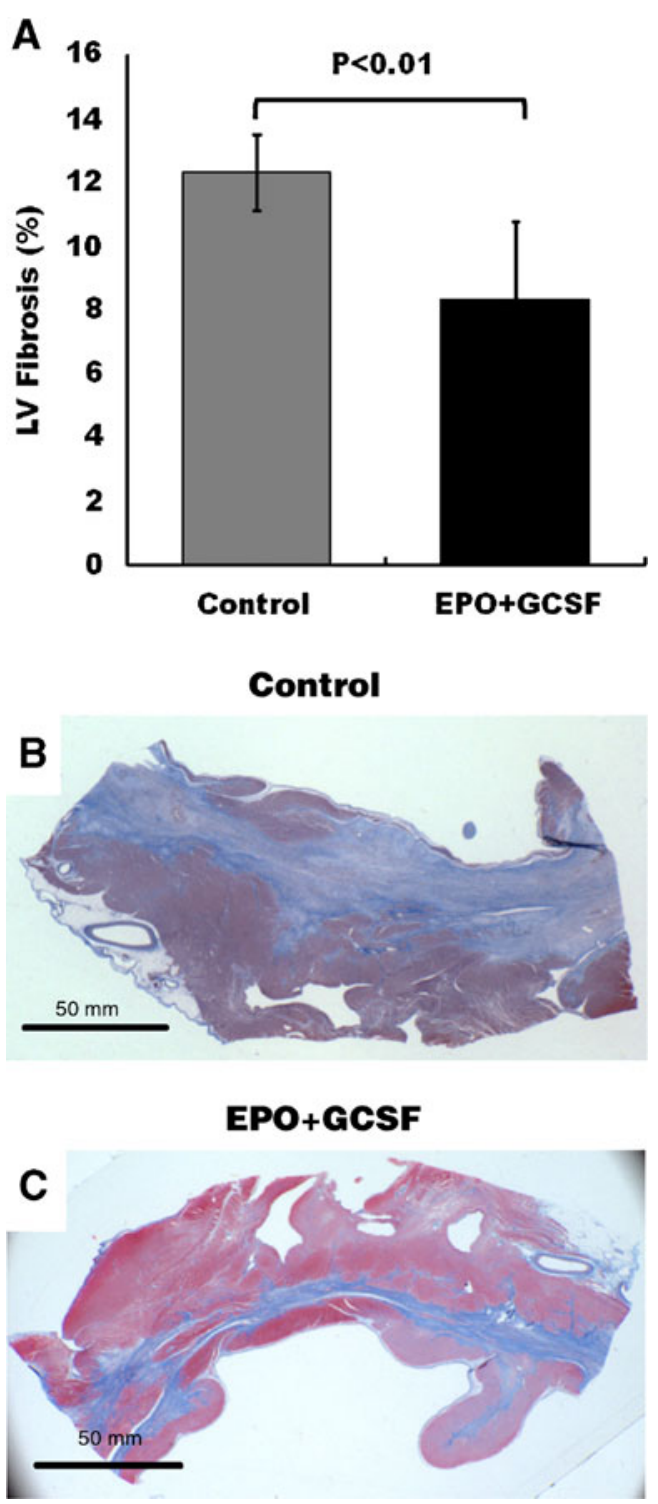

Fig. 5 Extent of fibrosis following myocardial infarction. a EPO+GCSF treatment is associated with decreased fibrosis of the left ventricle compared to the control group. Representative infarct zone regions (arc of the left ventricle containing scar tissue) stained with Masson Trichrome (fibrosis=blue) in sections of b Control, $\mathbf{c}$ EPO+GCSF. Data are mean \pm SD 
Infarct Border Zone
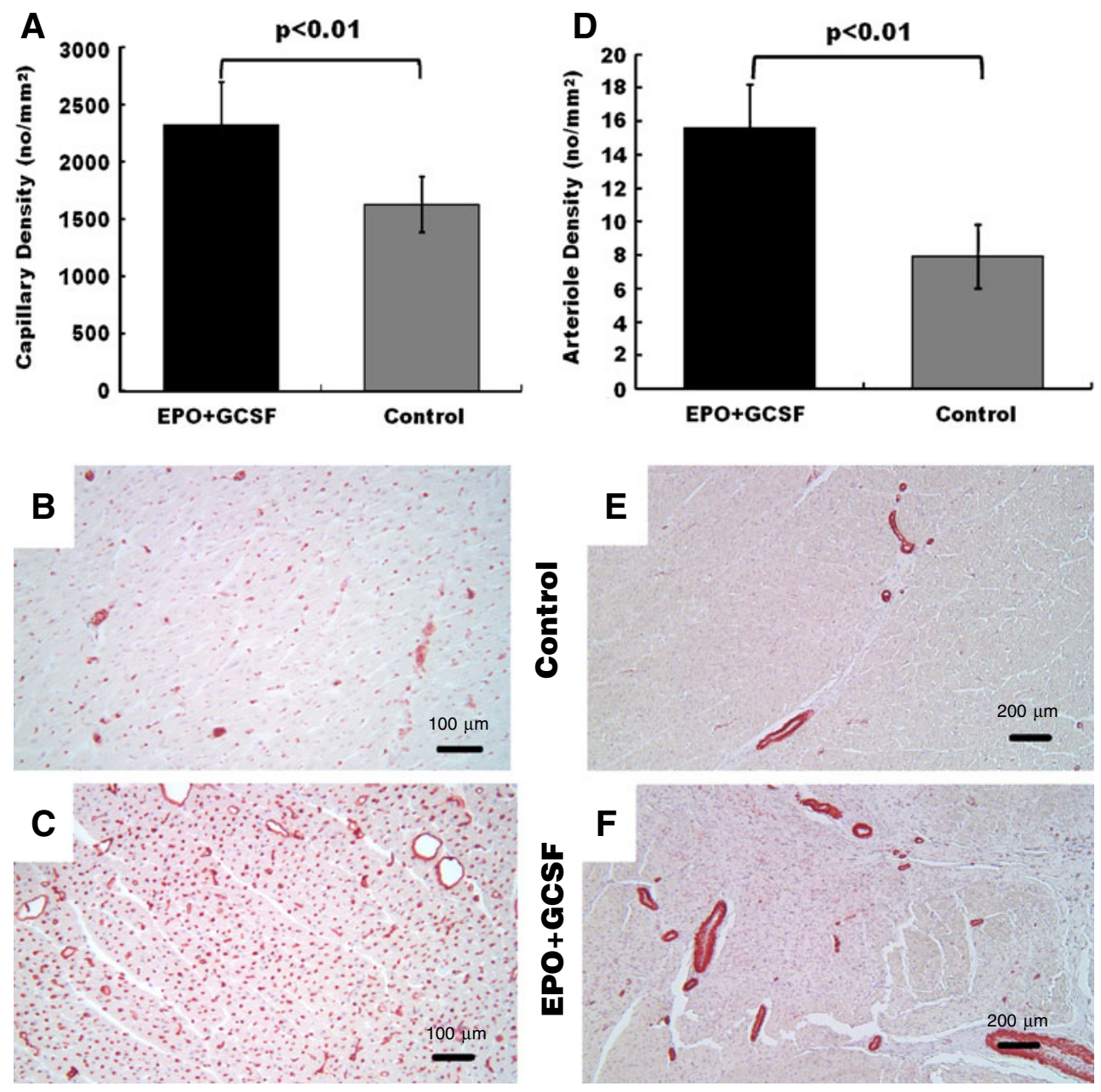

Fig. 6 Effect of cytokine therapy on vascular density. a EPO+GCSF therapy resulted in increased capillary density in the infarct border zone compared to control. Representative infarct border zone areas stained with antibodies against lectin (pink) in sections of $\mathbf{b}$ control vs. c EPO+GCSF. Scale bar $=200 \mu \mathrm{m}$. d EPO+GCSF results in increased

PRSW was significantly decreased at 1 weeks after MI in the control group compared to $\mathrm{EPO}+\mathrm{GCSF}$ therapy.

EPO + GCSF positively impacts diastolic function

As summarized in Table 2, peak negative $\mathrm{dP} / \mathrm{dt}$ was significantly lower in the EPO+GCSF group compared to the control. Also, the Tau constant was smaller in the EPO+GCSF group compared to the control pointing towards a beneficial effect of the $\mathrm{EPO}+\mathrm{GCSF}$ cytokine therapy on diastolic function.

Cytokine therapy leads to more viable myocardium

Measurements derived from gross images of serial myocardial slices suggested that compared with the control group, treatment with $\mathrm{EPO}+\mathrm{GCSF}$ did not lead to a

arteriole density at the infarct border zone compared to control. Representative infarct border zone areas stained with antibodies against smooth muscle actin (pink) in sections of e control vs. f EPO+GCSF treated pigs. Scale bar $=100 \mu \mathrm{m}$

reduction in the infarct size ( $16 \pm 4$ vs. $15 \pm 3 \%, p=$ ns). However, histological evaluation by Masson's Trichrome staining revealed an overall decrease in scar and fibrosis (Fig. 5a, $p<0.01$ ) in the EPO+GCSF group compared to the control, and therefore, more viable myocardium. The differences between the two techniques rely on the fact that the gross pathology evaluation does not take into account areas of viable tissue within the infarct zone, which are prevalent in this ischemia reperfusion model and contribute to the myocardial wall motion.

Cytokine combination therapy increases vascular density

As shown in Fig. 6a-c, capillary density at the infarct border zone was increased in $\mathrm{EPO}+\mathrm{GCSF}$ treated animals compared to control $(p<0.01)$ as was the arteriolar density $(p<0.01$, Fig. 6d-e). 
Table 3 Post hoc analyisnonsuperiority of combination versus monotherapy over time

The values are expressed as the mean \pm by standard error (in parentheses)

$L V E F$ left ventricle ejection fraction, LVEDV LV end-diastolic volume, LVESV LV end-systolic volume

NS Non significant

${ }^{\text {a }}$ Change from baseline value; significance of post-hoc test in repeated measures ANOVA design ${ }^{*} p<0.01$ vs. baseline, ${ }^{* *} p<0.05$ vs. 1 week, $* * * p<0.01$ vs. 1 week

\begin{tabular}{|c|c|c|c|c|}
\hline Parameter & $\mathrm{EPO} \pm \mathrm{GCSF}(n=8)$ & $\mathrm{EPO}(n=8)$ & $\operatorname{GCSF}(n=8)$ & ANOVA $F$ test \\
\hline \multicolumn{5}{|l|}{ LVEF (\%) } \\
\hline Baseline & $56.8(1.3)$ & $57.7(0.8)$ & $56.6(0.6)$ & NS \\
\hline 1 Week post $\mathrm{MI}^{\mathrm{a}}$ & $41.1(1.6)^{*}$ & $43.8(0.7)^{*}$ & $42.8(1.5)^{*}$ & NS \\
\hline 6 Weeks post $\mathrm{MI}^{\mathrm{a}}$ & $41(1.2)^{*}$ & $39.3(2.5)^{*}, * * *$ & $38.4(2.1)^{*}, * * *$ & NS \\
\hline $\begin{array}{l}\text { Repeated measures } \\
\text { ANOVA (main effect) }\end{array}$ & $p<0.01$ & $p<0.01$ & $p<0.01$ & \\
\hline \multicolumn{5}{|l|}{ LVEDV (mL) } \\
\hline Baseline & $53.2(0.9)$ & $53.2(2)$ & $49.6(1.2)$ & NS \\
\hline 1 Week post $\mathrm{MI}^{\mathrm{a}}$ & $62.5(1.5)^{*}$ & $63.5(2.5)^{*}$ & $60.7(2.1)^{*}$ & NS \\
\hline 6 Weeks post $\mathrm{MI}^{\mathrm{a}}$ & $67.9(2)^{*}, * *$ & $70.8(2.1)^{*}, * * *$ & $70.5(1.7)^{*}, * * *$ & NS \\
\hline $\begin{array}{l}\text { Repeated measures } \\
\text { ANOVA (main effect) }\end{array}$ & $p<0.01$ & $p<0.01$ & $p<0.01$ & \\
\hline \multicolumn{5}{|l|}{ LVESV (mL) } \\
\hline Baseline & $22.9(0.9)$ & $22.6(0.9)$ & $21.3(0.4)$ & NS \\
\hline 1 Week post $\mathrm{MI}^{\mathrm{a}}$ & $36.8(1.2)^{*}$ & $35.6(1.6)^{*}$ & $34.4(1)^{*}$ & NS \\
\hline 6 Weeks post $\mathrm{MI}^{\mathrm{a}}$ & $40.7(1.5)^{*}$ & $43.1(3.2)^{*}, * * *$ & $44(2.3)^{*}, * * *$ & NS \\
\hline $\begin{array}{l}\text { Repeated measures } \\
\text { ANOVA (main effect) }\end{array}$ & $p<0.01$ & $p<0.01$ & $p<0.01$ & \\
\hline
\end{tabular}

EPO+GCSF therapy increased the capillary density and arteriolar density at infarct zone compared to control (respectively, $1058 \pm 259$ vs. $508 \pm 56$ capillaries $/ \mathrm{mm}^{2}, p<$ $0.01 ; 27.5 \pm 6.8$ vs. $13.7 \pm 6$ arterioles $\left./ \mathrm{mm}^{2}, p<0.01\right)$. Capillary density at the infarct zone and LVEF at 6 weeks also showed significant correlation $(r=0.84 ; p<0.01)$.

Historical analysis shows non-superiority of EPO+GCSF combination therapy over monotherapy

In a historical analysis comparing monotherapy with EPO and GCSF recently published by our group [8, 15], and EPO+GCSF combination therapy, there was no significant differences in the LVEF and volumes overtime (Table 3). Therefore, although combination therapy seems to be safe and effective, it does not seem to be superior to monotherapy with either EPO or GCSF.

\section{Discussion}

The major findings of this study are: [1] EPO+GCSF combination therapy is safe; [2] EPO+GCSF stabilizes systolic function and prevents further deterioration of diastolic cardiac function post-MI; [3] EPO+GCSF therapy induces bone marrow cell mobilization; [4] EPO+GCSF leads to more viable myocardium and increased vascular density compared to the control group; [5] EPO+GCSF combination therapy does not seem to be superior than EPO or GCSF monotherapy in a historical post hoc analysis.

To our knowledge, this is the first report of EPO $+\mathrm{GCSF}$ combination therapy in the large animal acute MI model. In this study, we have used doses that our group tested as monotherapy in the same animal model [8, 15], and that have been approved for clinical use, demonstrating that combination therapy was safe and effective. The mild increase in the hemoglobin level at 4 weeks (peak of $13.8 \mathrm{~g} / \mathrm{dl}$ ) was not associated with thromboembolic events and the use of EPO was not associated with hypertension in the treated animals.

In our model, we found that EPO+GCSF combination therapy stabilized systolic function, and reduced post-MI remodeling by diminishing $\mathrm{LV}$ diastolic dilatation and pressures over time. Combination therapy also resulted in more viable myocardium and the better wall motion score corroborated this finding, demonstrating preservation of the wall motion at the infarcted area. In addition, EPO+GCSF combination therapy was associated with increased capillary and arteriolar density. This ability to promote revascularization may partly explain the results as previously demonstrated by us and others [12, 34, 35].

Interestingly, when we performed a historical comparison between combination therapy and our previous reports using EPO or GCSF as monotherapy [8, 15], all three therapeutic strategies were superior compared to the control arm. However, we could not demonstrate a clear and significant additive or synergistic effect on cardiac function with EPO+GCSF combination therapy over monotherapy.

EPO + GCSF combination therapy has proven useful in the treatment of patients with refractory anemia due to myelodysplastic syndrome by a synergistic inhibition of progenitor cell apoptosis, [36] and possibly by enhancement of stem cell mobilization [37]. We have recently evaluated the mobilization of Lin-/Sca-1+/c-kit+ cells from 
the bone marrow into the circulation post-MI in the eGFP+ chimeric mouse model, and demonstrated that combination of EPO+GCSF therapy resulted in significantly increased mobilization of $\mathrm{Lin}^{-} / \mathrm{Sca}-1^{+} / \mathrm{c}-\mathrm{kit}^{+}$cells into the circulation at 6 days post-MI compared with either EPO or GCSF monotherapy, or to control [38]. However, similar to this current report in the large animal MI model, EPO and GCSF combination therapy did not seem to have an additive benefit of combination therapy over monotherapy with either agent in the rodent MI model either [38].

There are a number of limitations that need to be pointed out in this study. Given the difficulties with housing large animals at our facility and the cost associated with such studies, the duration of follow-up and the number of animals/group had to be limited and only selected doses of the agents used could be studied. The follow-up time point in the current study was chosen based on previous pre-clinical investigations that demonstrated functional improvements by this time which plateau thereafter and to keep the design of the experiment consistent with our prior reports with the monotherapy arms $[8,15]$ to allow direct comparison [39]. We recognize that 6 weeks may be too short to encompass the complete evolution of cardiac remodeling and heart failure but longer study durations are very challenging to undertake in the large and growing porcine animal model.

In addition, the doses of these agents were chosen because of the safety profile in clinical settings. Clearly, different doses and combinations thereof could be studied but the cost of such a study in large animals would be prohibitive. Importantly, our study has limited ability to define the intrinsic mechanisms responsible for the improved cardiac function with $\mathrm{EPO}+\mathrm{GCSF}$ combination therapy vs. control. These detailed mechanistic questions are difficult to answer in large animal models and are outside the scope of this current report. Notably, we could not overcome the lack of well recognized porcine antibodies to characterize bone marrow progenitor cells both in the circulation and also in the heart. Finally, given the large size of the porcine hearts and the infarcted regions, accurate analyses for differential apoptosis are difficult to make in this model and as such were not undertaken in this study.

In conclusion, we report that prolonged combination therapy with $\mathrm{EPO}+\mathrm{GCSF}$ in a large animal model of acute MI with reperfusion has beneficial effects on left ventricular function and structure. EPO+GCSF combination therapy after acute MI led to an increase in viable myocardium, increased vascular density, and promoted stabilization of LV global function and improved indices of LV remodeling. In follow-up to a previous report from our laboratory using EPO or GCSF monotherapy post-MI in the large animal model, combination cytokine therapy with EPO+GCSF does not seem to be superior to monotherapy with either agent alone.
Acknowledgments We thank Gina Orcino for animal care and technical support, and Petros Minasi for administrative assistance.

This work was supported in part by the UCSF Cardiac Stem Cell Foundation (San Francisco, CA); a grant from the Wayne and Gladys Valley Foundation (Oakland, CA) and the C. Breetwor Foundation (Mountain View, CA). Darbepoetin (Aranesp) and GCSF (Neupogen) were provided by Amgen, Thousand Oaks, CA.

Open Access This article is distributed under the terms of the Creative Commons Attribution Noncommercial License which permits any noncommercial use, distribution, and reproduction in any medium, provided the original author(s) and source are credited.

\section{References}

1. Rosamond W, Flegal K, Furie K, et al. Heart disease and stroke statistics-2008 update: a report from the American heart association statistics committee and stroke statistics subcommittee. Circulation. 2008;117:e25-146.

2. Frantz S, Vallabhapurapu D, Tillmanns J, et al. Impact of different bone marrow cell preparations on left ventricular remodelling after experimental myocardial infarction. Eur J Heart Fail. 2008;10:119-24.

3. Dawn B, Guo Y, Rezazadeh A, et al. Postinfarct cytokine therapy regenerates cardiac tissue and improves left ventricular function. Circ Res. 2006;98:1098-105.

4. Piepoli MF, Vallisa D, Arbasi M, et al. Bone marrow cell transplantation improves cardiac, autonomic, and functional indexes in acute anterior myocardial infarction patients (Cardiac Study). Eur J Heart Fail. 2010;12:172-80.

5. Yeghiazarians Y, Zhang Y, Prasad M, et al. Injection of Bone Marrow Cell Extract Into Infarcted Hearts Results in Functional Improvement Comparable to Intact Cell Therapy. Mol Ther 2009;

6. Orlic D, Kajstura J, Chimenti S, et al. Mobilized bone marrow cells repair the infarcted heart, improving function and survival. Proc Natl Acad Sci U S A. 2001;98:10344-9.

7. Iwanaga K, Takano H, Ohtsuka M, et al. Effects of G-CSF on cardiac remodeling after acute myocardial infarction in swine. Biochem Biophys Res Commun. 2004;325:1353-9.

8. Angeli FS, Smith C, Amabile N, Shapiro M, Bartlett L, Virmani $\mathrm{R}$, et al. Granulocyte colony stimulating factor in myocardial infarction with low ejection fraction. Cytokine. 2010;51:278-85.

9. Abdel-Latif A, Bolli R, Zuba-Surma EK, Tleyjeh IM, Hornung CA, Dawn B. Granulocyte colony-stimulating factor therapy for cardiac repair after acute myocardial infarction: a systematic review and meta-analysis of randomized controlled trials. Am Heart J. 2008;156:216-26 e9.

10. Sterin-Borda L, Barcelo AC, Bozzini CE. Erythropoietin improves cardiac contractility in post-hypoxic mice. $\mathrm{Br} \mathrm{J}$ Haematol. 2003;121:180-6.

11. Rui T, Feng Q, Lei M, et al. Erythropoietin prevents the acute myocardial inflammatory response induced by ischemia/reperfusion via induction of AP-1. Cardiovasc Res. 2005;65:719-27.

12. Calvillo L, Latini R, Kajstura J, et al. Recombinant human erythropoietin protects the myocardium from ischemia-reperfusion injury and promotes beneficial remodeling. Proc Natl Acad Sci U S A. 2003;100:4802-6.

13. van der Meer P, Lipsic E, Henning RH, et al. Erythropoietin induces neovascularization and improves cardiac function in rats with heart failure after myocardial infarction. J Am Coll Cardiol. $2005 ; 46: 125-33$.

14. Lipsic E, Westenbrink BD, van der Meer P, van der Harst P, Voors AA, van Veldhuisen DJ. Low-dose erythropoietin improves cardiac 
function in experimental heart failure without increasing haematocrit. Eur J Heart Fail. 2008;10:22-9.

15. Angeli FS, Amabile N, Burjonroppa S, et al. Prolonged therapy with erythropoietin is safe and prevents deterioration of left ventricular systolic function in a porcine model of myocardial infarction. J Card Fail. 2010;16:579-89.

16. Ozaki K, Leonard WJ. Cytokine and cytokine receptor pleiotropy and redundancy. J Biol Chem. 2002;277:29355-8.

17. Mantovani A. The chemokine system: redundancy for robust outputs. Immunol Today. 1999;20:254-7.

18. Charo IF, Ransohoff RM. The many roles of chemokines and chemokine receptors in inflammation. N Engl J Med. 2006;354:610 21.

19. Angeli FS, Shapiro M, Amabile N, et al. Left ventricular remodeling after myocardial infarction: characterization of a swine model on beta-blocker therapy. Comp Med. 2009;59:272-9.

20. Lipsic E, van der Meer P, Voors AA, et al. A single bolus of a long-acting erythropoietin analogue darbepoetin alfa in patients with acute myocardial infarction: a randomized feasibility and safety study. Cardiovasc Drugs Ther. 2006;20:135-41.

21. Powell J, Gurk-Turner C. Darbepoetin alfa (Aranesp). Proc (Bayl Univ Med Cent). 2002;15:332-5.

22. Schiller NB, Shah PM, Crawford M, et al. Recommendations for quantitation of the left ventricle by two-dimensional echocardiography. American society of echocardiography committee on standards, subcommittee on quantitation of two-dimensional echocardiograms. J Am Soc Echocardiogr. 1989;2:358-67.

23. Kass DA, Midei M, Graves W, Brinker JA, Maughan WL. Use of a conductance (volume) catheter and transient inferior vena caval occlusion for rapid determination of pressure-volume relationships in man. Cathet Cardiovasc Diagn. 1988;15:192-202.

24. Grossman W, Braunwald E, Mann T, McLaurin LP, Green LH. Contractile state of the left ventricle in man as evaluated from end-systolic pressure-volume relations. Circulation. 1977;56:84552.

25. Sunagawa K, Maughan, W., Suga, H., Sugawa, K. Cardiac contraction and the pressure-volume relationship. Oxford: Oxford Univ.Press; 1988.

26. Baan J, Van der Velde ET. Sensitivity of left ventricular endsystolic pressure-volume relation to type of loading intervention in dogs. Circ Res. 1988;62:1247-58.

27. Baan J, van der Velde ET, de Bruin HG, et al. Continuous measurement of left ventricular volume in animals and humans by conductance catheter. Circulation. 1984;70:812-23.
28. Raff GL, Glantz SA. Volume loading slows left ventricular isovolumic relaxation rate. Evidence of load-dependent relaxation in the intact dog heart. Circ Res. 1981;48:813-24.

29. Glower DD, Spratt JA, Snow ND, et al. Linearity of the FrankStarling relationship in the intact heart: the concept of preload recruitable stroke work. Circulation. 1985;71:994-1009.

30. Amado LC, Schuleri KH, Saliaris AP, et al. Multimodality noninvasive imaging demonstrates in vivo cardiac regeneration after mesenchymal stem cell therapy. J Am Coll Cardiol. 2006;48:2116-24.

31. Cerqueira MD, Weissman NJ, Dilsizian V, et al. Standardized myocardial segmentation and nomenclature for tomographic imaging of the heart: a statement for healthcare professionals from the cardiac imaging committee of the council on clinical cardiology of the American heart association. Circulation. 2002;105:539-42.

32. Kass DA, Maughan WL. From 'Emax' to pressure-volume relations: a broader view. Circulation. 1988;77:1203-12.

33. Nordhaug D, Steensrud T, Korvald C, Aghajani E, Myrmel T. Preserved myocardial energetics in acute ischemic left ventricular failure - studies in an experimental pig model. Eur J Cardiothorac Surg. 2002;22:135-42.

34. de Boer RA, Pinto YM, Suurmeijer AJ, et al. Increased expression of cardiac angiotensin II type 1 (AT(1)) receptors decreases myocardial microvessel density after experimental myocardial infarction. Cardiovasc Res. 2003;57:434-42.

35. Sugano Y, Anzai T, Yoshikawa T, et al. Granulocyte colonystimulating factor attenuates early ventricular expansion after experimental myocardial infarction. Cardiovasc Res. 2005;65:446-56.

36. Balleari E, Rossi E, Clavio M, et al. Erythropoietin plus granulocyte colony-stimulating factor is better than erythropoietin alone to treat anemia in low-risk myelodysplastic syndromes: results from a randomized single-centre study. Ann Hematol. 2006;85:174-80.

37. Rigolin GM, Porta MD, Ciccone $\mathrm{M}$, et al. In patients with myelodysplastic syndromes response to rHuEPO and G-CSF treatment is related to an increase of cytogenetically normal CD34 cells. Br J Haematol. 2004;126:501-7.

38. Yeghiazarians Y, Khan M, Angeli FS, et al. Cytokine combination therapy with long-acting erythropoietin and granulocyte colony stimulating factor improves cardiac function but is not superior than monotherapy in a mouse model of acute myocardial infarction. J Card Fail. 2010;16:669-78.

39. Fuchs S, Baffour R, Zhou YF, et al. Transendocardial delivery of autologous bone marrow enhances collateral perfusion and regional function in pigs with chronic experimental myocardial ischemia. J Am Coll Cardiol. 2001;37:1726-32. 